\title{
Determinación de parámetros cinéticos por la técnica calorimétrica de barrido único. Aplicación a la cristalización no isoterma de la aleación $\mathrm{Sb}_{0.12} \mathrm{As}_{\mathbf{0 . 4 0}} \mathrm{Se}_{0.48}$
}

\author{
J. VÁZQUEZ, P.L. LÓPEZ-ALEMANY, P. VILLARES Y R. JIMÉNEZ-GARAY. \\ Departamento de Física de la Materia Condensada. Facultad de Ciencias. Universidad de Cádiz. 11510. Puerto Real (Cádiz).
}

\begin{abstract}
Se ha desarrollado un procedimiento para analizar la evolución temporal de la fracción cristalizada y calcular los parámetros cinéticos de reacciones no isotermas que implican la formación y crecimiento de núcleos. Considerando las hipótesis de volumen extendido y nucleación al azar, se ha obtenido una expresión general de la fracción transformada como función del tiempo en procesos de cristalización isotermos. La velocidad de cristalización deducida se ha aplicado a procesos no isotermos bajo la restricción de nucleación al principio de la transformación y que se anula posteriormente. En estas condiciones, se han deducido los parámetros cinéticos usando la técnica de barrido único. El método teórico desarrollado se ha aplicado a la cristalización de la aleación semiconductora $\mathrm{Sb}_{0.12} \mathrm{As}_{0.40} \mathrm{Se}_{0.48}$. Los parámetros cinéticos obtenidos difieren alrededor del $6 \%$ de los calculados por técnicas de barrido múltiple, lo que confirma la fiabilidad y exactitud de la técnica de barrido único cuando se calculan los mencionados parámetros en procesos no isotermos.
\end{abstract}

Palabras clave: Nucleación y crecimiento cristalino, calorimetría diferencial de barrido, técnica termoanalítica de barrido único, velocidad de calentamiento, parámetros cinéticos.

Determination of kinetic parameters by means the calorimetric technique of single-scan. Application to the non-isothermal crystallization of the $\mathrm{Sb}_{0.12} \mathrm{As}_{0.40} \mathrm{Se}_{0.48}$ alloy

A procedure has been developed for analyzing the evolution with time of the crystallized fraction and for calculating the kinetic parameters at non-isothermal reactions involving formation and growth of nuclei. Considering the assumptions of extended volume and random nucleation, a general expression of the transformed fraction as a function of time has been obtained in isothermal crystallization processes. The crystallization rate deduced has been applied to the non-isothermal processes under the restriction of a nucleation which takes place early in the transformation and the nucleation frequency is zero thereafter. In these conditions, the kinetic parameters have been deduced by using the technique of single-scan. The theoretical method developed has been applied to the crystallization kinetics of the semiconducting $\mathrm{Sb}_{0.12} \mathrm{As}_{0,40} \mathrm{Se}_{0.48}$ alloy. The kinetic parameters obtained differ by only about $6 \%$ of those calculated by multiple-scan techniques, which confirms the reliability and accuracy of the single-scan technique when calculating the mentioned parameters in non-isothermal processes.

Keywords: Nucleation and crystal growth, differential scanning calorimetry, thermo-analytical technique of single-scan, heating rate, kinetic parameters.

\section{INTRODUCCIÓN}

Aunque el vidrio se ha usado como medio artístico y material industrial durante siglos, sin embargo, la "ciencia del vidrio" no surge como un campo de investigación floreciente hasta épocas relativamente recientes. En las últimas décadas se ha registrado un fuerte interés teórico y práctico por la aplicación de técnicas experimentales de análisis isotermas y no isotermas al estudio de las transformaciones vidrio-cristal. Si bien, las técnicas isotermas de análisis son más definitivas, en la mayoría de los casos, sin embargo, las técnicas termoanalíticas no isotermas tienen varias ventajas. La rapidez con que pueden realizarse los experimentos no isotermos los hace altamente interesantes. Muchas transformaciones de fase transcurren demasiado rápidamente para medirlas en condiciones isotermas, debido a los transitorios inherentemente asociados con el dispositivo experimental. Los procesos industriales dependen, con frecuencia, del comportamiento cinético de sistemas que experimentan transformaciones de fase en condiciones no isotermas. Desde este punto de vista, es muy intere- sante obtener medidas precisas de las cinéticas de transformaciones no isotermas.

Las experiencias descritas en la literatura $(1,2)$ indican que la velocidad de crecimiento de los cristales en los líquidos, que forman vidrios, no está limitada por la eliminación de calor de la superficie de separación cristal-líquido. Desde este punto de vista, los líquidos que forman vidrios pueden proporcionar sistemas únicos para apreciar la validez de los modelos teóricos usados en la descripción de procesos de crecimiento cristalino. Los mencionados líquidos proporcionan sistemas en los que la temperatura de la superficie cristal-líquido está bien definida por la temperatura del sistema y, por consiguiente, la evolución temporal de la cinética de cristalización puede medirse adecuadamente usando técnicas de análisis térmico. Debido a esto, las experiencias de calorimetría diferencial de barrido (DSC) y análisis térmico diferencial (DTA) se consideran, en la actualidad, técnicas aplicables al estudio de transformaciones de fase que implican nucleación y crecimiento crista- 
lino, en particular a la cinética de cristalización de líquidos que forman vidrios (3-6). Con muy pocas excepciones, el análisis de los datos obtenidos se realiza mediante la ecuación de la velocidad de transformación de Johnson-Mehl-Avrami (JMA) $(7,8)$.

En este trabajo se establecen las condiciones de aplicabilidad de la ecuación de JMA a procesos de cristalización no isotermos y se deducen sus parámetros cinéticos mediante la técnica de barrido único. Finalmente, se aplica la técnica citada al análisis de la cinética de cristalización de la aleación vítrea $\mathrm{Sb}_{0.12} \mathrm{As}_{0.40} \mathrm{Se}_{0.48}$ y los valores de los parámetros así obtenidos difieren alrededor del $6 \%$ de los calculados por técnicas de barrido múltiple. Este hecho muestra la fiabilidad y exactitud de la técnica de barrido único cuando se calculan los mencionados parámetros a partir de tratamientos de calentamiento continuo.

\section{TEORIA}

La cristalización es un caso particular de las transformaciones de estado sólido controladas por nucleación y crecimiento de grano. Si un embrión de la fase transformada nuclea en el instante $\tau$ y después crece, en general, anisotrópicamente con velocidades principales de crecimiento, $u_{\mathrm{i}}\left(t^{\prime}\right)(\mathrm{i}=1,2,3)$, en tres direcciones mutuamente perpendiculares, su volumen $\mathrm{v}$ en el instante $t\left(\tau<t^{\prime}<t\right)$ es

$$
v(\tau, t)=g \prod_{i} \int_{\tau}^{t} u_{\mathrm{i}}\left(t^{\prime}\right) \mathrm{d} t^{\prime}
$$

donde $g$ es un factor geométrico dependiente de la forma del crecimiento cristalino y la expresión

$$
\prod_{\mathrm{i}} \int_{\tau}^{t} u_{\mathrm{i}}\left(t^{\prime}\right) \mathrm{d} t^{\prime}
$$

condensa el producto de las integrales correspondientes a los valores del subíndice i.

Cuando se desprecia la solapación de granos se puede definir un volumen extendido, $V_{\mathrm{e}^{\prime}}$ mediante la relación

$$
\mathrm{d} V_{\mathrm{e}}=v(\tau, t) I_{V}(\tau)\left(V_{\mathrm{a}}+V_{\mathrm{b}}\right) \mathrm{d} \tau=v(\tau, t) V I_{V}(\tau) \mathrm{d} \tau
$$

siendo

$I_{V}(\tau)$ la frecuencia de nucleación por unidad de volumen, $V_{\mathrm{a}} \mathrm{y}$ $V_{\mathrm{b}}$ los volúmenes no transformado y transformado, respectivamente, y $V$ el volumen de la muestra completa. La variación del volumen real transformado, $\mathrm{d} V_{\mathrm{b}^{\prime}}$ y la del volumen extendido, $\mathrm{d} V_{\mathrm{e}^{\prime}}$ están relacionados por

$$
\mathrm{d} V_{\mathrm{b}}=\left(1-V_{\mathrm{b}} V^{-1}\right) \mathrm{d} V_{\mathrm{e}}=(1-x) \mathrm{d} V_{\mathrm{e}}
$$

siendo $x=V_{\mathrm{b}} V^{-1}$ la fracción transformada. Diferenciando esta expresión y substituyendo el resultado en la ec.[3] se obtiene

$$
(1-x)^{-1} \mathrm{~d} x=V^{-1} \mathrm{~d} V_{\mathrm{e}}
$$

ecuación que se relaciona con la ec.[2], donde se incluye la expresión de $v(\tau, t)$ dada en la ec.[1], resultando

$$
x(t)=1-\exp \left\{-g \int_{0}^{t} I_{V}(\tau)\left[\prod_{\mathrm{i}} \int_{\tau}^{t} u_{\mathrm{i}}\left(t^{\prime}\right) \mathrm{d} t^{\prime}\right] \mathrm{d} \tau\right\}
$$

la ecuación básica de nucleación y crecimiento para la fracción transformada $x(t)$.

Cuando la velocidad de crecimiento es isotrópica, $u_{\mathrm{i}}\left(t^{\prime}\right)=u\left(t^{\prime}\right)$, hipótesis de acuerdo con la evidencia experimental, la ec.[5] puede escribirse como

$$
x(t)=1-\exp \left\{-g \int_{0}^{t} I_{V}(\tau)\left[\int_{\tau}^{t} u\left(t^{\prime}\right) \mathrm{d} t^{\prime}\right]^{m} \mathrm{~d} \tau\right\}
$$

donde $m$ es un exponente que depende del mecanismo de crecimiento y de la dimensionalidad del cristal.

En el caso de cristalización isoterma con $I_{V} \mathrm{y} u$ independientes del tiempo la integración de la ec.[6] da

$$
x(t)=1-\exp \left(-g^{\prime} I_{V} u^{m} t^{n}\right)
$$

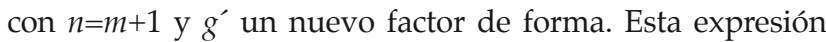
puede considerarse como un caso específico de la ecuación de transformación de JMA

$$
x(t)=1-\exp \left(-K t^{n}\right)
$$

En esta ecuación, K es la constante de velocidad de reacción, función de la temperatura y $n$ es un parámetro que refleja la frecuencia de nucleación y / o la morfología del crecimiento. La velocidad de transformación isoterma se deduce derivando con respecto al tiempo la ec.[8] y substituyendo en la expresión resultante la forma explícita de $t$ a partir de la ec.[8] dando

$$
\mathrm{d} x / \mathrm{d} t=n K^{1 / n}(1-x)[-\ln (1-x)]^{(n-1) / n}
$$

expresión conocida como ecuación de velocidad de transformación de JMA.

\subsection{Evaluación de la ecuación de velocidad de JMA en con- diciones no isotermas}

La deducción de las ecs.[8], [9] se basa en las siguientes hipótesis:

1. Condiciones de transformación isoterma.

2. Nucleación distribuida espacialmente al azar.

3. Velocidad de crecimiento de la nueva fase dependiente sólo de la temperatura y no del tiempo, es decir, "cinética de crecimiento lineal".

Por tanto, de acuerdo con la literatura (9), la ec.[9] sólo puede aplicarse a transformaciones no isotermas en un número limitado de casos. En particular, si la velocidad de transformación sólo depende de las variables de estado, fracción transformada y temperatura, entonces la ec.[9] puede usarse para describir transformaciones no isotermas. Bajo estas restricciones y asignando a la constante de velocidad de reacción una dependencia de la temperatura tipo Arrhenius ( $K=K_{0} \exp (-$ $E / R T)$ ), los parámetros cinéticos asociados con la ecuación de velocidad de JMA son: la energía de activación efectiva, $E$, el exponente cinético, $n$, y el factor de frecuencia, $K_{0}$. Dichos parámetros pueden evaluarse utilizando la técnica de análisis de barrido único. 


\subsubsection{TÉCNICA DE BARRIDO ÚNICO}

La deducción de relaciones para calcular los parámetros cinéticos de transformaciones de estado sólido usando técnicas no isotermas, requiere una velocidad de reacción independiente de la historia térmica y que pueda expresarse como el producto de dos funciones separables de $T$ y $x$, respectivamente. En estas condiciones la ec.[9] puede escribirse

$$
\mathrm{d} x / \mathrm{d} t=n K^{1 / n} f(x)=n K_{0}^{1 / n} f(x)[\exp (-E / n R T)]
$$

Teniendo en cuenta que la velocidad de calentamiento es $\beta=$ $\mathrm{d} T / \mathrm{d} t$, la ec.[10] se integra separando las variables, resultando

$$
\int_{0}^{x} \frac{\mathrm{d} x^{\prime}}{\left(1-x^{\prime}\right)\left[-\ln \left(1-x^{\prime}\right)\right]^{(n-1) / n}}=\frac{n K_{0}^{1 / n}}{\beta} \int_{T_{0}}^{T} e^{-E / n R T^{\prime}} \mathrm{d} T^{\prime}
$$

y usando las substituciones $z^{\prime}=-\ln \left(1-x^{\prime}\right)$ e $y^{\prime}=E / n R T^{\prime}$, la ec. [11] se transforma en

$$
[-\ln (1-x)]^{1 / n}=K_{0}^{1 / n} E(n \beta R)^{-1} \int_{y}^{\infty} e^{-y^{\prime}} y^{\prime-2} \mathrm{~d} y^{\prime}=K_{0}^{1 / n} E(n \beta R)^{-1} I
$$

La integral $I$ se evalúa mediante la serie alternada (10)

$$
-e^{-y^{\prime}} y^{\prime-2} \sum_{k=0}^{w}(-1)^{k}(k+1) ! y^{\prime-k}
$$

donde se pueden tomar sólo los dos primeros términos, sin cometer error apreciable, y obtener

$$
I=\left(\frac{n R T}{E}\right)^{2}\left(1-\frac{2 n R T}{E}\right) \exp (-E / n R T)
$$

Substituyendo esta expresión de $I$ en la ec.[12], y tomando logaritmo en la función resultante, se obtiene

$$
\ln [-\ln (1-x)]-2 n \ln T=-\frac{E}{R T}+n \ln \frac{n R K_{0}^{1 / n}}{\beta E}
$$

si se

admite que el término $2 n R T$ / $E$ en la ec.[13] es despreciable en comparación con la unidad, usualmente $E / R T \geq 25$ (11). Cuando se conoce $n$, la gráfica de $\ln [-\ln (1-x)]-2 n \ln T$ frente a $1 / T$ puede ajustarse a una recta, cuya pendiente da un valor para la energía de activación. No obstante, de acuerdo con la literatura (11), en un rango de temperatura de $100 \mathrm{~K}$ puede despreciarse la contribución del término $2 n \ln T$ sin cometer error apreciable en la $E$ calculada. Además, tomando logaritmo en la ec.[10] resulta

$$
\ln (\mathrm{d} x / \mathrm{d} t)=-\frac{E}{n R T}+\ln [f(x)]+\ln \left(n K_{0}^{1 / n}\right)
$$

ecuación de una recta cuya pendiente permite obtener $E / n$, si se considera que para $0.25<x<0.75$ la función $\ln [f(x)]$ es prácticamente constante (11). Finalmente, conocidos los parámetros $E$ y $n$, se obtiene el factor de frecuencia, $K_{0}$, a partir de la ec.[10].

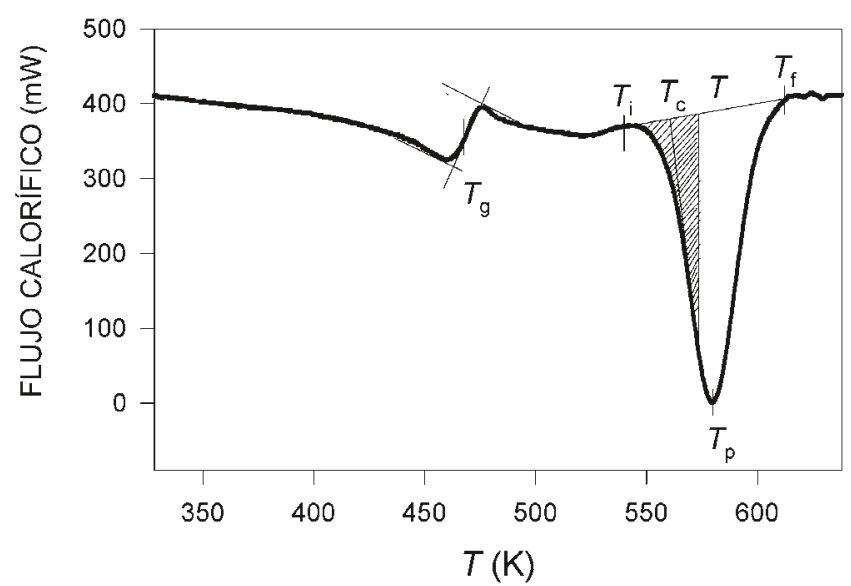

Fig.1. Gráfica DSC típica de la aleación $\mathrm{Sb}_{0.12} \mathrm{As}_{0.40} \mathrm{Se}_{0.48}$ para una velocidad de calentamiento de $8 \mathrm{~K} \mathrm{~min}^{-1}$. La zona rayada muestra el área, $A_{T}$, entre $T_{\mathrm{i}} \mathrm{y} T$.

\section{PROCEDIMIENTO EXPERIMENTAL}

La aleación semiconductora $\mathrm{Sb}_{0.12} \mathrm{As}_{0.40} \mathrm{Se}_{0.48}$ se obtuvo en forma másica a partir de sus elementos del $99.999 \%$ de pureza, que se introdujeron en una ampolla de cuarzo sellada a una presión de $10^{-2} \mathrm{~N} \mathrm{~m}^{-2}$. Esta ampolla se mantuvo en un horno rotatorio a $1225 \mathrm{~K}$ durante $24 \mathrm{~h}$. y luego se enfrió bruscamente en un baño de agua para evitar la cristalización del material. La naturaleza vítrea del mismo se comprobó con un barrido de rayos-X en un difractómetro Siemens D500 y su composición y homogeneidad se verificó mediante microscopía electrónica de barrido en un microscopio Jeol JSM-820. El comportamiento térmico se investigó usando un calorímetro diferencial de barrido Perkin-Elmer DSC7 con precisión de $\pm 0.1 \mathrm{~K}$. Los datos cinéticos se tomaron a partir de barridos de las muestras desde la temperatura ambiente a velocidades de calentamiento de 2 , 4, 8, 16, 32 y $64 \mathrm{Kmin}^{-1}$. La temperatura de transición vítrea se tomó en el punto de inflexión de la curva termográfica, como se ve en la Fig.1. La fracción cristalizada, $x$, a una temperatura, $T$, puede expresarse como $x=A_{T} / A$, donde $A$ es el área limitada por la mencionada curva entre la temperatura inicial, $T_{\mathrm{i}}$, y la final, $T_{\mathrm{f}}$ del proceso de cristalización y $A_{T}$ es el área entre $T_{\mathrm{i}} \mathrm{y}$ una temperatura genérica, $T$, como muestra la Fig.1.

\section{RESULTADOS Y DISCUSIÓN}

La cinética de cristalización de la aleación $\mathrm{Sb}_{0.12} \mathrm{As}_{0.40} \mathrm{Se}_{0.48}$ se ha analizado, de acuerdo con la literatura (11), considerando una velocidad de crecimiento cristalino con dependencia de la

TABLA I. TEMPERATURAS CARACTERÍSTICAS Y ENTALPÍAS DEL PROCESO DE CRISTALIZACIÓN DE LA ALEACIÓN $\mathrm{Sb}_{0.12} \mathrm{As}_{0.40} \mathrm{Se}_{0.48}$

\begin{tabular}{|c|c|}
\hline Parámetros & Valores experimentales \\
\hline$T_{\mathrm{g}}(\mathrm{K})$ & $459.2-490.9$ \\
$T_{\mathrm{i}}(\mathrm{K})$ & $534.7-579.8$ \\
$T_{\mathrm{p}}(\mathrm{K})$ & $579.3-641.4$ \\
$\Delta T(\mathrm{~K})$ & $51.1-73.0$ \\
$\Delta H\left(\mathrm{mcal} \mathrm{mg}^{-1}\right)$ & $4.1-5.1$ \\
\hline
\end{tabular}


temperatura tipo Arrhenius y frecuencia de nucleación despreciable, es decir, la condición de "saturación de posiciones" (12). Se ha aplicado la ecuación de JMA bajo condiciones no isotermas utilizando la técnica de barrido único. En la Tabla I se dan los intervalos de variación de las magnitudes obtenidas de los termogramas registrados a las velocidades de calentamiento, citadas en la Sección 3. En dicha tabla, $T_{\mathrm{g}}$ es la temperatura de transición vítrea, $T_{\mathrm{p}}$ es la correspondiente a la máxima $\mathrm{d} x / \mathrm{d} t$, $\Delta T$ es la anchura del pico de cristalización y $\Delta H$ la entalpía del proceso. Los cocientes entre las ordenadas de los termogramas y las áreas de los picos dan las correspondientes velocidades de cristalización, que permiten construir las curvas representadas en la Fig.2. La técnica de barrido único se ha aplicado a conjuntos de datos experimentales a distintas $\beta$. En el caso de los conjuntos de valores de las magnitudes $T$ y $x$ en los intervalos $(604.5-613.5) \mathrm{K}$ y $(0.2-0.5)$ respectivamente, correspondientes a $\beta=16 \mathrm{~K} \mathrm{~min}^{-1}$, la Fig. 3 muestra la gráfica de $\ln [-\ln (1-$ $x$ )] frente a $1 / T$ (ec.[14]) y la recta de regresión correspondiente, de cuya pendiente se ha obtenido una energía de activación de $76.6 \mathrm{kcal} \mathrm{mol}^{-1}$. Además usando un conjunto de valores de $\mathrm{d} x / \mathrm{d} t$ en el intervalo $\left(6.82 \times 10^{-3}-9.21 \times 10^{-3}\right) \mathrm{s}^{-1}$, se ha obtenido la recta de regresión, $\ln (\mathrm{d} x / \mathrm{d} t)=-19146.2 \times T^{-1}+26.68$, representada en la Fig.4. La pendiente de esta recta, de acuerdo con la ec.[15], da $E / n=38.3 \mathrm{kcal} \mathrm{mol}^{-1}$ y la ordenada en el origen, $K_{0}=2.33 \times 10^{23} \mathrm{~s}^{-1}$. A partir de los valores de $E$ y $E / n$ se obtiene inmediatamente el exponente cinético, $n=2$.

Con objeto de confirmar la fiabilidad del método descrito se han evaluado también los parámetros cinéticos mediante otros tratamientos matemáticos citados en la literatura (13-17). Como ejemplo se presenta el cálculo de dichos parámetros mediante el método de Kissinger (15). Por tanto usando la relación lineal

$$
\ln \left(T_{\mathrm{p}}^{2} / \beta\right)=E / n R T_{\mathrm{p}}-\ln \left(n R K_{0}^{1 / n} / E\right)
$$

se puede obtener el cociente $E / n$ y el factor de frecuencia $K_{0}$. Para ello se han representado en la Fig.5 los valores experimentales de $\ln \left(T_{p}{ }^{2} / \beta\right)$ frente a $1 / T_{p}$ y la recta de regresión correspondiente. La pendiente y la ordenada en el origen de la citada recta dan $E / n=38.5 \mathrm{kcal} \mathrm{mol}^{-1}$ y $K_{0}=1.31 \times 10^{22} \mathrm{~s}^{-1}$, respectivamente. Además, los datos experimentales $T_{\mathrm{p}} \mathrm{y}(\mathrm{d} x / \mathrm{d} t){ }_{p^{\prime}}$ recogidos en la Tabla II, permiten obtener para cada velocidad de calentamiento, mediante la relación,

$$
E=\left.R T_{\mathrm{p}}^{2}(\mathrm{~d} x / \mathrm{d} t)\right|_{\mathrm{p}}(0.37 \beta)^{-1}
$$

la energía de activación del proceso, cuyos valores se dan también en la Tabla II, siendo su valor medio $<E>=72.4 \mathrm{kcal}$

TABLA II. VELOCIDAD DE CRISTALIZACIÓN MÁXIMA, TEMPERATURA CORRESPONDIENTE Y ENERGÍA DE ACTIVACIÓN PARA DIFERENTES VELOCIDADES DE CALENTAMIENTO.

\begin{tabular}{|c|c|c|c|}
\hline$\beta\left(\mathrm{K} \mathrm{min}^{-1}\right)$ & $T_{\mathrm{p}}(\mathrm{K})$ & $\left.10^{3}(\mathrm{~d} x / \mathrm{d} t)\right|_{\mathrm{p}}\left(\mathrm{s}^{-1}\right)$ & $E\left(\mathrm{kcal} \mathrm{mol}^{-1}\right)$ \\
\hline 2 & 579.3 & 1.37 & 74.4 \\
4 & 587.0 & 2.48 & 69.4 \\
8 & 601.4 & 5.18 & 76.0 \\
16 & 610.9 & 9.80 & 74.2 \\
32 & 625.8 & 18.26 & 72.5 \\
64 & 641.4 & 32.43 & 67.7 \\
\hline
\end{tabular}

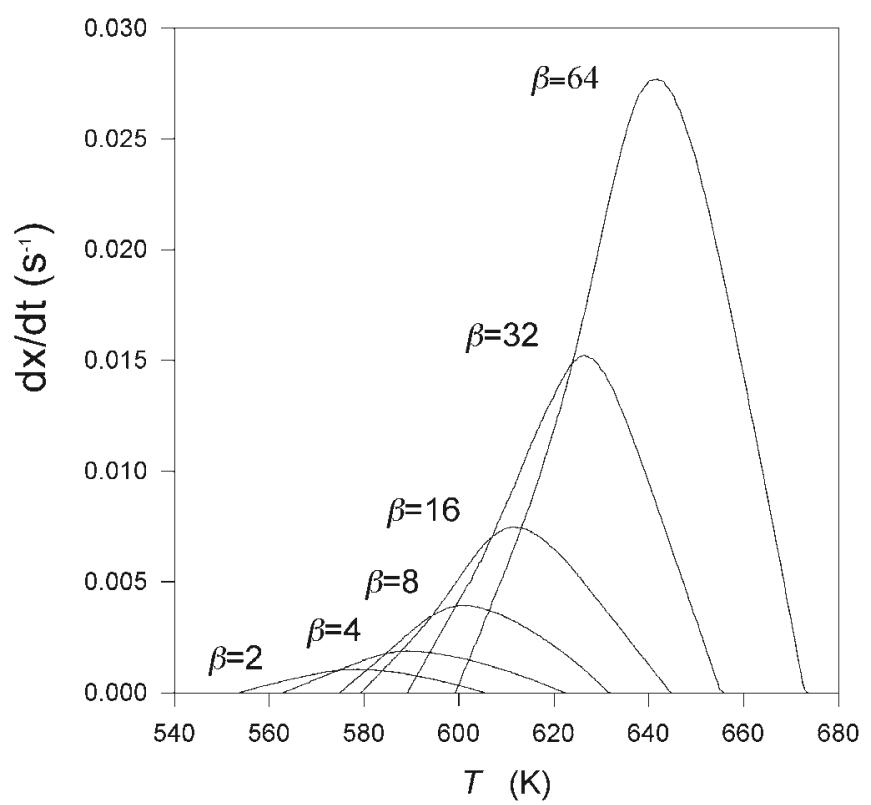

Fig.2. Velocidad de cristalización frente a la temperatura a diferentes velocidades de calentamiento.

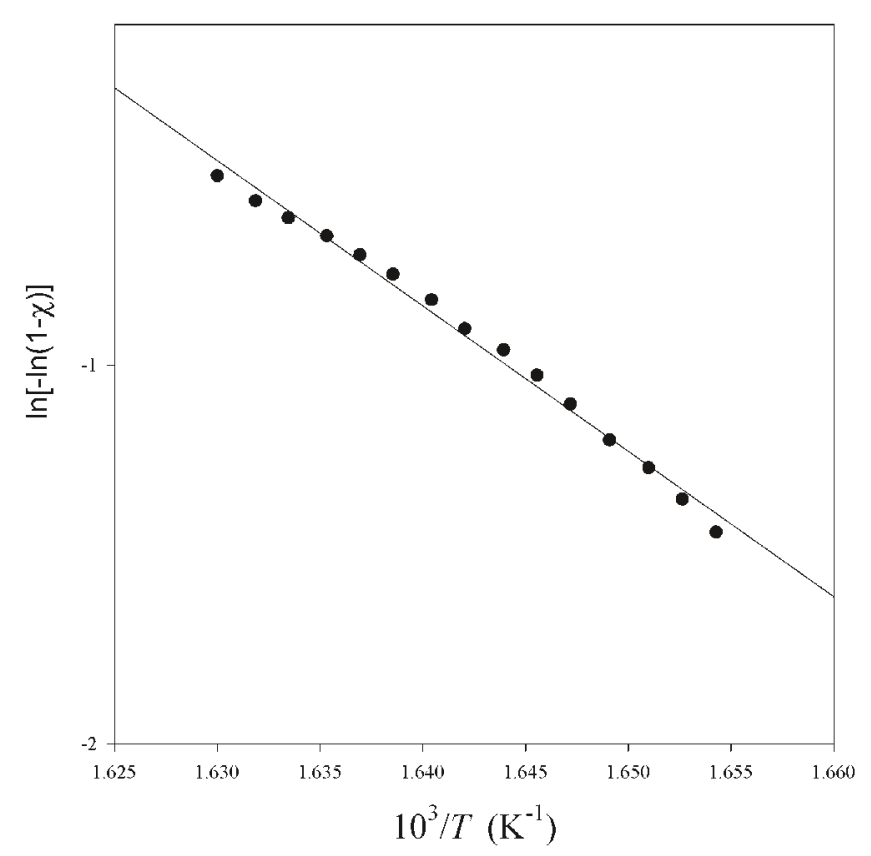

Fig.3. Variación de $\ln [-\ln (1-x)]$ con $1 / T$ para $\beta=16 \mathrm{~K} \mathrm{~min}^{-1}$. 


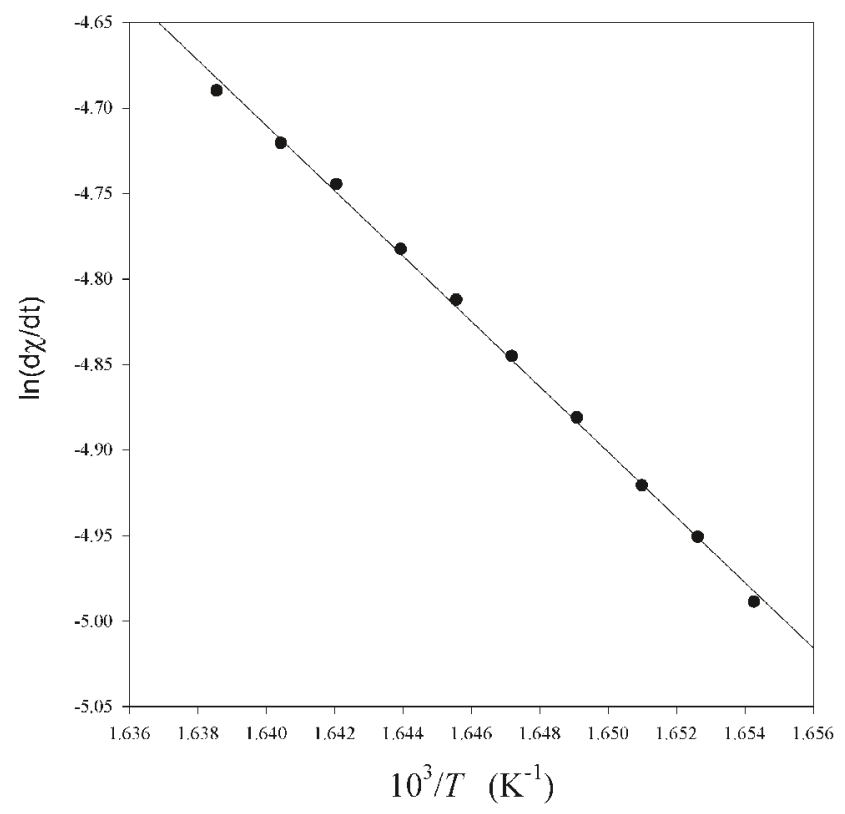

Fig.4. Representación de $\ln (\mathrm{d} x / \mathrm{d} t)$ frente a $1 / T$ y recta de regresión correspondiente para la aleación $\mathrm{Sb}_{0.12} \mathrm{As}_{0.40} \mathrm{Se}_{0.48}$.

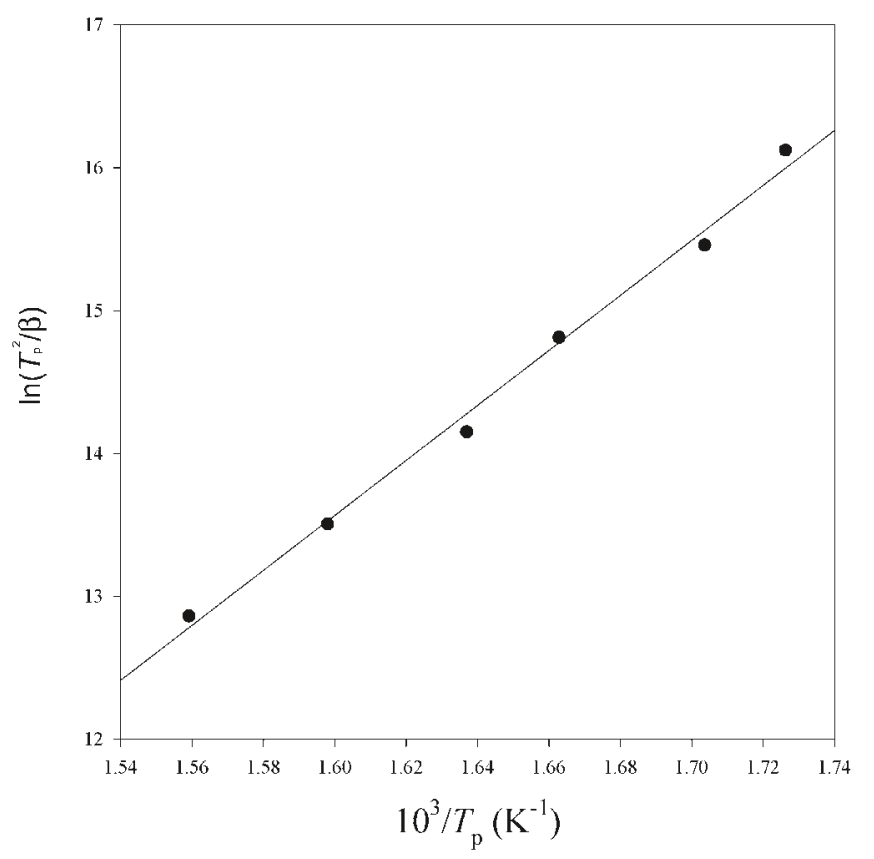

Fig.5. Gráfica de $\ln \left(T_{\mathrm{p}}^{2} / \beta\right)$ frente $1 / T_{\mathrm{p}}$ del material analizado $\left(\beta\right.$ en $\left.\mathrm{K} \mathrm{s}^{-1}\right)$. $\mathrm{mol}^{-1}$. Una vez conocidos $E / n$ y $E$ se ha obtenido inmediatamente el exponente cinético $n=1.88$. Se han comparado los resultados de los parámetros $E, n$ y $\ln K_{0}$ evaluados por este método y los obtenidos con la técnica de barrido único, encontrándose que el error entre ellos para el parámetro menos preciso es inferior al 6\%. Este resultado está de acuerdo con la literatura (11), donde se afirma que para $(n-1) / n=0.6$ en el rango de $0.2<x<0.5$ resulta un error del $7 \%$ en la energía de activación, valor aceptable en la mayoría de las reacciones de cristalización.

\section{CONCLUSIONES}

El método descrito permite estudiar la evolución temporal de la fracción transformada en materiales, que implican procesos de nucleación y crecimiento cristalino. Este método se basa en el concepto de volumen extendido en el material transformado, y en la condición de nucleación al azar. Partiendo de estas suposiciones se ha deducido una expresión general de la fracción transformada como función del tiempo. En el caso de cristalización isoterma se ha transformado la citada expresión en una ecuación, que puede tomarse como un caso específico de la ecuación de transformación de JMA. La aplicación de esta ecuación a transformaciones no isotermas supone condiciones restrictivas, como es el caso de la velocidad de transformación dependiente únicamente de la fracción transformada y de la temperatura. Bajo esta restricción se han obtenido los parámetros cinéticos, usando la técnica de barrido único, que puede aplicarse a procesos de cristalización en materiales, que cumplen la ecuación de velocidad de JMA.

La técnica mencionada se ha aplicado a la cinética de cristalización de la aleación vítrea $\mathrm{Sb}_{0.12} \mathrm{As}_{0.40} \mathrm{Se}_{0.48}$. La diferencia entre los valores obtenidos para los parámetros cinéticos por esta técnica, y los calculados por el método de Kissinger es inferior al $6 \%$. Este buen acuerdo pone de manifiesto la fiabilidad de la técnica de barrido único para calcular los parámetros cinéticos, principalmente en el intervalo (0.2-0.5) de fracción cristalizada, un hecho que está de acuerdo con la literatura.

\section{AGRADECIMIENTOS}

Los autores agradecen a la Junta de Andalucía y a la CICYT su apoyo financiero (Proyecto $n^{\circ}$ MAT98-0791)

\section{BIBLIOGRAFÍA}

1. G.S. Meiling and D.R. Uhlmann, "Crystallization and Melting Kinetics of Sodium Disilicate". Phys. Chem. Glasses 8 62-68 (1967).

2. P.J. Vergano and D.R. Uhlmann, "Crystallization Kinetics of Germanium Dioxide: Effect of Stechiometry on Kinetics". Phys. Chem. Glasses 11 30-38 (1970).

3. D. Clinton, R.A. Mercer and R.P. Miller, "Application of Differential Thermal Analysis by High-Temperature Microscopy to Kinetic and Structural Studies on the Crystallization of Glasses". J. Mat. Sci. 5 171-181 (1970).

4. D.D. Thornburg and R.I. Johnson, “Thermal Analysis of Bulk Amorphous Arsenic Triselenide". J. Non-Cryst. Solids 17 2-8 (1975).

5. N. Clavaguera, "Crystallization Kinetics of a Se-Ge-Sb Alloy Glass". J. Non-Cryst. Solids 22 23-26 (1976).

6. H.S. Chen, "A Method for Evaluating Viscosities of Metallic Glasses from the Rates of Thermal Transformations". J. Non-Cryst. Solids 27 257-263 (1978).

7. W.A. Johnson and R.F. Mehl, "Reaction Kinetics in Processes of Nucleation and Growth". Trans. Am. Inst. Min. Engrs 135 416-442 (1939). 
8. M. Avrami, "Granulation, Phase Change, and Microstructure". J. Chem. Phys. 9 177-184 (1941).

9. T. Kemény, "The Evaluatión of Kinetic Parameters from Non-Isotermal Experiments". Thermochim. Acta 110 131-134 (1987).

10. J. Vázquez, C. Wagner, P. Villares and R. Jiménez-Garay, "A Theoretical Method for Determining the Crystallized Fraction and Kinetic Parameters by DSC, Using Non-Isothermal Techniques". Acta Mater. 44 4807-4813 (1996).

11. H. Yinnon and D.R. Uhlmann, "Applications of Thermoanalytical Techniques to the Study of Crystallization Kinetics in Glass-Forming Liquids". J. Non-Cryst. Solids 54, 253-275 (1983).

12. J. Vázquez, P.L. López-Alemany, P. Villares and R. Jiménez-Garay, "A Study on the Thermal Stability of some Alloys in the Cu-Ge-Te Glassy System by Differential Scanning Calorimetry". Thermochim. Acta 327 191-196 (1999).

13. Y.Q. Gao, W. Wang, F.Q. Zheng and X. Liu, "On the Crystallization
Kinetics of $\mathrm{Pd}_{80} \mathrm{~B}_{4} \mathrm{Si}_{16}$ Glass". J. Non-Cryst. Solids 81 135-139 (1986).

14. J.A. Augis and J.E. Bennett, "Calculation of the Avrami Parameters for Heterogeneous Solid State Reactions Using a Modification of the Kissinger Method". J. of Therm. Anal. 13 283-292 (1978).

15. H.E. Kissinger, "Reaction Kinetics in Differential Thermal Analysis". Analyt. Chem. 29 1702-1706 (1957).

16. J. Vázquez, P.L. López-Alemany, P. Villares and R. Jiménez-Garay, “A Study on Non-Isothermal Transformation Kinetics. Application to the Crystallization of $\mathrm{Sb}_{0.20} \mathrm{As}_{0.32} \mathrm{Se}_{0.48}$ Alloy". J. of Alloys and Compounds 270 179-185 (1998).

17. J. Vázquez, C. Wagner, P. Villares and R. Jiménez-Garay, "A Study of the Glass Forming Ability of some Alloys in the Ge-As-Te System by Differential Scanning Calorimetry". Mater. Chem. Phys 58 187-190 (1999). 